\title{
Finite Element Analysis of Damage Evolution of Micro-Holes in Complex Shape of Automotive Casting Aluminum Alloy
}

\author{
Ping Zhang 1,2,a , Xiao Yu 2,b , Yuanyuan $\mathrm{Li}^{2, \mathrm{c}}$, Limei Wang ${ }^{1, \mathrm{~d}}$ Youqiang Wang ${ }^{1, \mathrm{e},{ }^{*}}$ \\ ${ }^{1}$ School of Mechanical Engineering, Qingdao University of Technology, Shandong Qingdao, \\ 266520.China \\ 2School of Mechanical Engineering, Qingdao Huanghai University, Qingdao 266033, China \\ a18661660729@163.com, b434463230@qq.com, c 568146084@qq.com, \\ d841086061@qq.com ,e'wyq1970301@126.com
}

Keywords: Complex shape micropores; Finite element model; Nucleation; Growth

\begin{abstract}
In this paper, the three-dimensional finite element model of single and double micropores with complex shape of cast aluminum alloy was established based on ABAQUS. The three dimensional finite element model of single and double micropores was established by using Johnson-Cook constitutive model. The finite element simulation of the penetration behavior of micro - holes with single complex shape micropores is carried out. The simulation results show that the maximum stress appears at the tip of the micropores under the external load, and the micro-cracks are induced at the tip of the micro-pits with the nucleation and growth of the micropores.The maximum stress appears in the radial maximum size of the cavity wall and the dimple tip of the micropores. Under the action of the external load, the deformation of the double-hole micropores increases with the symmetry of the double- Center formed through
\end{abstract}

\section{Introduction}

With the continuous progress of science and technology, industrial production is facing light weight. Aluminum alloy with low density, but the strength is relatively high, close to or more than high-quality steel, plastic good, can be processed into a variety of profiles, with excellent conductivity, thermal conductivity and corrosion resistance, etc., is the preferred lightweight structural materials, And has been widely used in automobiles, aerospace, etc. [1,2]. Considering the phenomenon of inclusions in the casting process, the sum of the shrinkage of the liquid and the solidification shrinkage in the casting process will produce different shrinkage (micropores) in the matrix [3]. Shrinkage and casting speed, the higher the casting speed, the more likely to produce shrinkage, regardless of the pouring speed to what extent, shrinkage damage is unavoidable, and the shape of the shrinkage are arbitrary [4]. With the development of aluminum industry, domestic and foreign experts and scholars on the aluminum alloy hole and crack defects carried out a lot of research. At the end of the 20th century, Zheng Changqing et al. [5] analyzed the conventional model of hole growth (R-T model and Gurson model), and established a new model (combination model). Li Zhenhuan [6] and others through the spherical single hole growth, nucleation and damage evolution of the finite element analysis, obtained spherical micro-hole damage evolution law. In foreign countries, experts and scholars have done a lot of research on the nucleation of holes, and Needleman and Tvergarrd [7] and others through numerical analysis of multiple microvoids, found that: with the constant change in hybrid, micro when the hole volume fraction reaches a critical value, the phenomenon of hole penetration occurs. Brock and Sun [8] et al. found that the nucleation of micropores was dependent on the tip of the hole. Although domestic and foreign experts and scholars on the micro-hole research increased year by year, but most of the micro-hole shape to simplify, it does not reflect the micro-hole defects in the real situation [9]. In this paper, casting aluminum alloy as the object of study, the design of complex shape of the micro-hole defects, the use of ABAQUS6.11 software for its finite element analysis, the complex shape of micro-hole damage evolution law. 


\section{Finite element modeling}

The finite element model established in this paper is shown in Fig. The shape of the internal microvoid defect is designed as spherical micropores. Spherical micro pits at the center of the specimen.

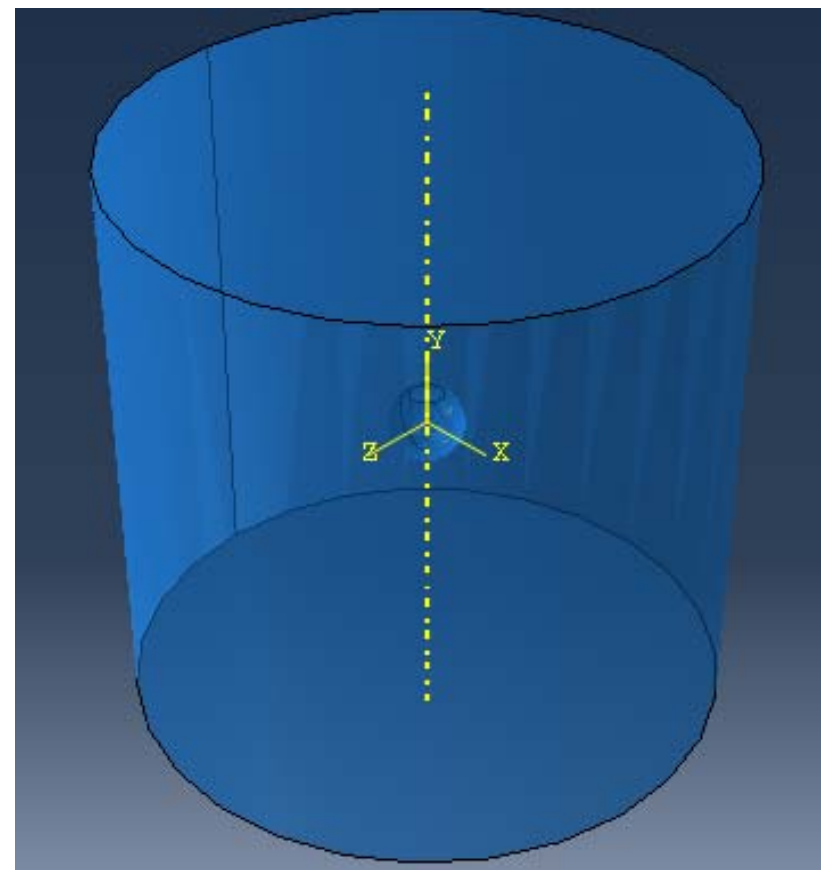

Fig. 1 The finite element model of single holes

\section{Material constitutive model}

The constitutive model describing the properties of materials is numerous, and the Johnson-Cook model is widely used in this paper. Johnson-Cook constitutive model is described as follows [10].

$$
\bar{\sigma}=\left(A+B \varepsilon^{n}\right)\left(1+C \ln \left(\frac{\dot{\varepsilon}}{\dot{\varepsilon}_{0}}\right)\right)\left(1-\left(\frac{T-T_{\text {room }}}{T_{\text {melt }}-T_{\text {room }}}\right)^{m}\right)
$$

Where $\mathrm{A}, \mathrm{B}, \mathrm{C}, \mathrm{n}, \mathrm{m}$ are the basic parameters that reflect the softness of the material strength, plasticity and temperature. The specific parameters are obtained by dynamic impact test and Original data fitting, as shown in Table 1.

Table1. material parameters of Johnson-Cook Model

\begin{tabular}{ccccc}
\hline $\mathrm{A} / \mathrm{Mpa}$ & $\mathrm{B} / \mathrm{Mpa}$ & $\mathrm{n}$ & $\mathrm{c}$ & $\mathrm{m}$ \\
\hline 542.24 & 273 & 0.194 & 0.136 & 0.271 \\
\hline
\end{tabular}

\section{Finite element analysis}

\subsection{Finite Element Analysis of Single Holes}

Figure 2 depicts the stress cloud of a single spherical dimple hole. Figure 2 (a) - (d) are not loaded, loaded the initial stage, stable deformation stage and growth stage.It can be seen from Fig. 2 that the single micropores will grow up in the load direction when they are loaded. At the initial load stage, the maximum stress of the micropores is the largest and the symmetrical distribution is distributed. And the micropores are elongated [11]; stress concentration occurs at the tip of the pits; when the micropores grow up to a certain extent, the micropores are cracked at the tip of the micropores until they are destroyed. 


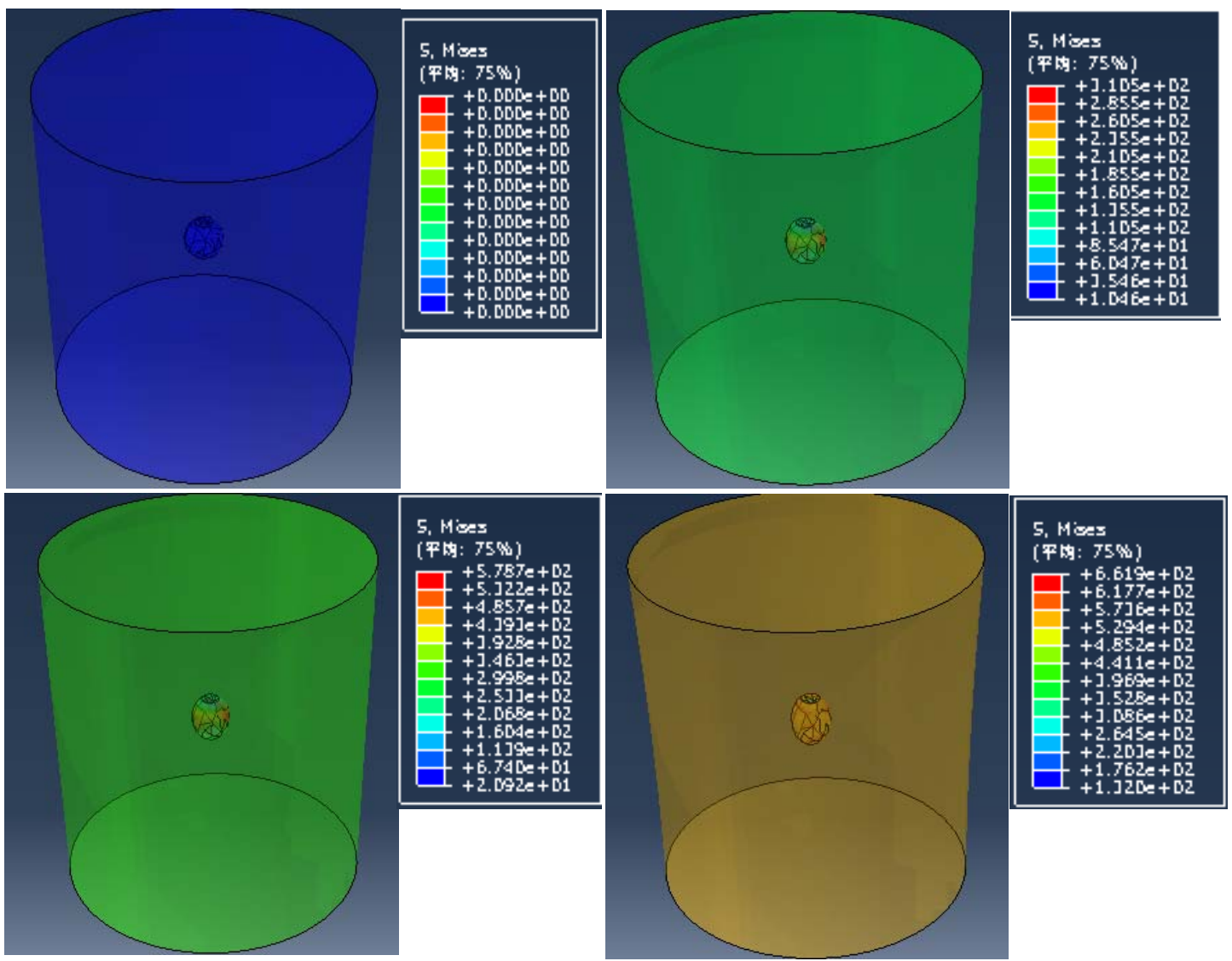

Fig.2 stress nephogram of single hole

\subsection{Finite element analysis of porous holes}

Figure 3 shows the finite element model of the double hole, and the shape of the internal microvoid defect is still designed as spherical micropores. The spherical micropores are located at the center axis symmetry and centroid.

Figure 4 shows the stress cloud of the double spherical microlevel hole. Figure 2(a)-(d), respectively, for the nucleation, growth and porous through the different stages.

It can be seen from Fig. 4 that the double-spherical micro-pit hole in the hole when the load, the same load along the direction of the phenomenon of growth; with the micro-hole size gradually grow, the connection between the two holes strength, A certain degree of weakening, until the last show two holes through the trend or phenomenon [12]. The maximum stress of the micropores in Fig. 4 occurs at the cavity of the cavity with the largest void size or at the tip of the pit. The increase in stress at the tip of the pit leads to the generation of microstructures. The reason is that the position of the two microvoids And the shape is completely symmetrical, the two micro-pits are directly opposite, increasing the strain energy of the material at the junction, stimulating the generation of microcracks, and finally making the two holes penetrate [13], resulting in the overall strength of the material falling until the damage. 


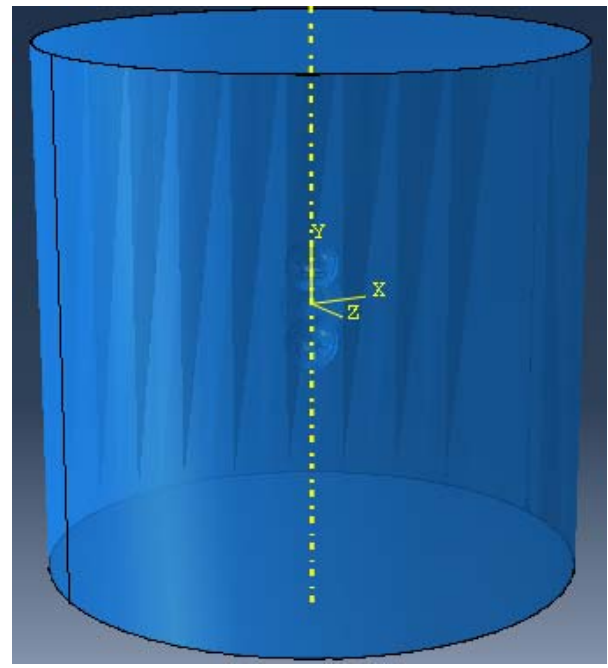

Fig.3 The finite element model of double holes

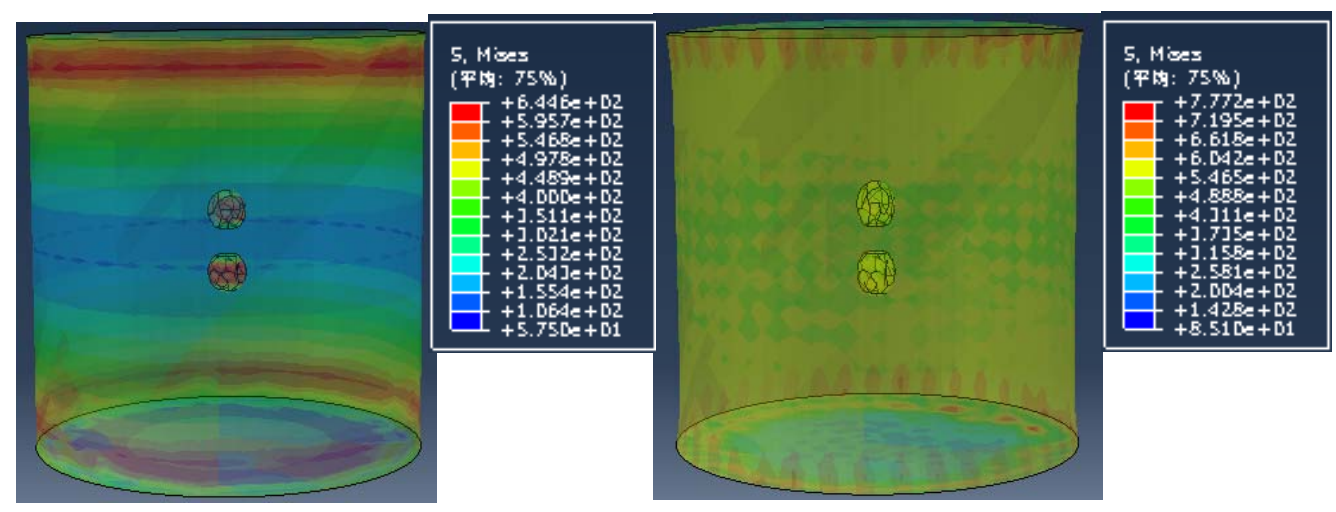

Fig. 4 stress nephogram of double hole

\section{Conclusion}

(1) Under the external load, the maximum stress appears at the tip of the micro-pits, and the micro-cracks are induced at the tip of the micro-pits with the nucleation and growth of the micropores.

(2) Under the external load, the maximum stress appears in the radial cavity of the inner cavity and the dimple tip of the micro-pits. With the increase of the symmetrical double-hole deformation, The two holes are formed at the center.

\section{Acknowledgements}

This paper is supported by the National Natural Science Foundation of China (51575289); Shandong Provincial Natural Science Foundation of China (ZR2016EEP03); Shandong University Science and Technology Program (J17KA031)

\section{References}

[1]. WAN Qian, ZHAO Hai-dong, ZOU Chun.Three-dimensional characteristics and distribution of micro-holes in aluminum alloy die casting [J] .Acta Metallurgica Sinica, 2013,03: 284-290.Journal of Huazhong University of Science and Technology (Natural Science Edition), 2012, S2: 64-67.

[2]. Jiang Wenming, Fan Zitian. 
[3]. HAN Zhi-qiang, LI Jin-xi, YANG Wen, ZHAO Hai-dong, LIU Bai-cheng Modeling and Simulation of Micro-hole Formation in Extrusion Casting Process of Aluminum Alloy [J] .Acta Metallurgica Sinica, 2011,01: 7-16.

[4]. Wan Qian, Zhao Haidong, Ge Ji-long.Effects of microvoids on fatigue properties of aluminum alloy die castings [J]. Journal of Nonferrous Metals, 2015,03: 568-574.

[5]. Zheng Changqing et al. Preliminary study and application of ductile fracture micromechanics [M]. Xi'an: Northwestern Polytechnical University Press, 1988 (Zheng Changqing et al. The Primary Study and Its Application of Ductile Meso-Mechanics. Xi'an : Northwestern Polyechnic University Press, 1988 (in Chinese))

[6]. Li Zhenhuan, Kuang Zhenbang.Study on the Polymerization Mechanism and Effective Energy Criterion of Holes Considering Three Dimensional Constraints[J]Acta Mechanica Sinica,2000, (4).

[7]. Tvergarrd V, Needleman A. Analisys of the cup-conc fracture in a round tensile bar [J]. Scta Meter, 1984, 32: 157 169

[8]. Brocks W, Sun DZ, Hönign A. Verificaion of the transferability of micromechanical parameters by cell model calculations with bisco-plastic materials [J]. Int J of Plasticity, 1995, 11 (8): 971 989

[9]. D. dela Fuente, E. Otero-Huerta, M. Morcillo. Studies of long-term weathering of aluminum in the atmosphere [J]. Corrosion Science, 2007, 49: $3134 \sim 3148$.

[10]. LIAO HL, LIN JC, LEE S L. Effect of pre-immersion on the SCC of heat-treated AA7050 in an alkaline 3.5\% Na Cl [J]. Corrosion Science, 2009,51 (2): $209 \sim 216$ The

[11]. Bengtesson BD, Svensson JE, Johansson L G. The influence of $\mathrm{CO} 2$, AlCl3 • 6H2O, $\mathrm{MgCl} 2 \bullet$ $6 \mathrm{H} 2 \mathrm{O}, \mathrm{Na} 2 \mathrm{SO} 4$ and $\mathrm{NaCl}$ on the atmospheric corrosion [J]. Corrosion Science, 2006, 48: 1848-1866.

[12]. Gao Meng, Xu Peng. High impact under the foam aluminum filling structure crushing energy absorption [J]. Ordnance Material Science and Engineering, 2016,01: 1-4.

[13]. HU Xiao-yan, XU Jian-gang, YAN Xin-xing.Molecular dynamics simulation of single crystal aluminum hole growth [J]. Journal of Materials Science and Engineering, 2012,06: 52-55. 